\title{
Visual acuity is correlated with ischemia and neurodegeneration in patients with early stages of diabetic retinopathy
}

\author{
Jin Li ${ }^{1}$, Yue Zhou ${ }^{1,3}$, Feng Chen ${ }^{1}$, Yingzi Li ${ }^{1}$, Rong Zhou ${ }^{1}$, Chaoming Wu ${ }^{2}$, Huankai Yu ${ }^{1}$, Zhiyang Lin ${ }^{1}$, Ce Shi ${ }^{1}$, \\ Gu Zheng ${ }^{1}$, Yilei Shao ${ }^{1}$, Qi Chen ${ }^{1}$, Fan Lu ${ }^{1 *+}$ and Meixiao Shen ${ }^{1 *+}$ (D)
}

\begin{abstract}
Purpose: We investigated the effects of retinal ischemia, neurodegeneration, and subclinical edema on best-corrected visual acuity (BCVA) in the early stages of diabetic retinopathy (DR).

Methods: Ischemia was evaluated by the microvascular parameters measured by optical coherence tomography angiography. Neurodegeneration and subclinical edema were identified by the intraretinal layer thickness obtained by optical coherence tomography. Eyes with nonproliferative diabetic retinopathy $(n=132)$ from 89 patients were analyzed. Eyes were classified as having normal BCVA $(n=88$ [66.7\%], Snellen equivalent $\geq 20 / 20)$ or decreased BCVA $(n=44$ [33.3\%], Snellen equivalent $<20 / 20$ ). The prevalence of ischemia, neurodegeneration, and subclinical edema was explored in patients with and without decreased BCVA, and correlations between BCVA and these pathological pathways were determined.

Results: Vessel density in the deep retinal capillary plexus (DRCP) and thickness of ganglion cell layer plus inner plexiform layer (GCL-IPL) were significantly lower in eyes with decreased BCVA compared with eyes with normal BCVA (both $P<0.05$ ). In the final multiple regression predictive model, age, DRCP vessel density, and GCL-IPL thickness (all $P \leq 0.044)$ were predictors of BCVA. DRCP vessel density and GCL-IPL thickness have an interactive effect on visual acuity. The proportions of ischemia and neurodegeneration were significantly higher in eyes with decreased BCVA than in eyes with normal BCVA ( $P=0.001$ and $P=0.004$, respectively).
\end{abstract}

Conclusion: During the natural course of the early stages of $D R$, ischemia and neurodegeneration were the main disease pathways associated with visual acuity, and the mechanisms varied among patients.

Keywords: Diabetic retinopathy, Visual acuity, Optical coherence tomography, Ischemia, Neurodegeneration

\section{Background}

Diabetic retinopathy (DR) is a leading cause of vision impairment and blindness among working adults worldwide [1]. In recent years, DR has been characterized by the predominance of one of the three phenotypes:

\footnotetext{
*Correspondence: lufan62@mail.eye.ac.cn; smx77@sohu.com

${ }^{\dagger} \mathrm{Fan}$ Lu and Meixiao Shen contributed equally to this work

${ }^{1}$ School of Ophthalmology and Optometry, Wenzhou Medical University, 270 Xueyuan Road, Wenzhou 325027, Zhejiang, China

Full list of author information is available at the end of the article
}

ischemia, neurodegeneration, and edema [2-4]. These three phenotypes vary greatly among different diabetic patients and lead to different risks for development of vision-threatening complications [5]. In view of the relevance of DR as a public health issue and a huge financial burden to government agencies, insurance companies, and individuals [6], it is important to improve our understanding of the mechanisms of the three phenotypes and the roles they play in the vision loss that occurs during the initial stages of DR. This information may help to 
develop improved early and individualized intervention strategies.

DR is conventionally described as a microvasculopathy; thus, the gold standard of severity classification is also based on this concept [7], and greater levels of DR severity usually correspond to worse vision loss. However, this correlation is very weak in nonproliferative diabetic retinopathy (NPDR) $[8,9]$. This indicates that microvascular changes are not the only pathological factor of the early visual impairment, and other mechanisms or metabolic changes are also implicated. In addition, many studies have indicated that ischemic and microvascular changes may lead to neurodegeneration or macular edema [10]. It is unclear if there are any correlations of the ischemic, neurodegenerative, and edematous phenotypes with vision loss. Consequently, there is no information regarding any potential interaction of these phenotypes that can affect visual acuity in the early stage of DR in patients who have no apparent macular complications. This is because commonly measured parameters, such as the size of the foveal avascular zone (FAZ) assessed by fluorescein angiography and whole retinal thickness assessed by optical coherence tomography (OCT) are of limited use for characterizing ischemia, neurodegeneration, and edema present in the early stages of DR.

Recently, investigators have used OCT with a sublayer segmentation algorithm to quantify the neurodegeneration characterized by the thinning of retinal nerve fiber layer (RNFL) and ganglion cell layer plus inner plexiform layer (GCL-IPL) [11-13]. OCT can also detect subclinical edema that is not observed clinically. It is diagnosed based on the thickness of the inner nuclear layer (INL), outer plexiform layer (OPL), and total retina that exceed the normal values [14]. In addition, ischemia can be identified by decreased vessel density and increased FAZ using optical coherence tomography angiography (OCTA) [15-17]. Therefore, OCT and OCTA offer the possibility of quantitatively assessing the associations among the three phenotypes with the loss of visual acuity in the initial stages of DR. The goal of our study was to use OCT and OCTA to quantify three pathological alterations, i.e., ischemia, neurodegeneration, and subclinical edema in the early stages of type 2 DR and to explore the correlations with visual acuity.

\section{Methods}

\section{Subject data collection}

Of the 89 patients with type 2 diabetes mellitus (DM) who participated in this study, 69 were enrolled from the Endocrinology Department of the Second Affiliated Hospital of Wenzhou Medical University, Wenzhou, China, where they were diagnosed by an endocrine specialist $(\mathrm{CW})$. Twenty patients were enrolled from the
Fundus Department at the Eye Hospital of Wenzhou Medical University. To serve as a control reference group for comparison with the study group of DR patients, an additional 33 healthy subjects ( 21 women and 12 men; $56.9 \pm 7.3$ years) were recruited from workers at the Eye Hospital of Wenzhou Medical University and family members of patients at the same hospital. With the exception of having DR, the inclusion and exclusion criteria were the same as for the DR patients. This study followed the tenets of the Declaration of Helsinki and was approved by the ethics committee of the Eye Hospital of Wenzhou Medical University. Written informed consent was obtained from all patients.

All subjects underwent a series of ophthalmologic examinations, including slit-lamp biomicroscopy, intraocular pressure (IOP) measurement and ophthalmoscopy. After a full objective and subjective refraction, the best-corrected distance visual acuity was determined monocularly in logarithm of the minimum angle of resolution (logMAR). The DR severity level was determined independently by two experienced graders and was based on the 7-field protocol using the ETDRS classification. Only patients with type 2 DM and NPDR were enrolled. Potential subjects and controls were excluded if they had lens or other ocular media opacities preventing detailed imaging, high myopia (under -6.00 diopters), clinical evidence of any other maculopathy, glaucoma or other neurodegenerative conditions, or any previous treatment of DR. Patients with a current or previous history of diabetic macular edema, defined as a central subfield thickening of at least $275 \mu \mathrm{m}$ on spectral domain OCT (SD-OCT) [18], were also excluded. Demographic information collected from the patients included age, sex, body mass index, mean arterial pressure, duration of $\mathrm{DM}$, and fasting blood glucose.

Based on best-corrected visual acuity (BCVA) levels, eyes of the diabetic cohort were divided into two groups: eyes with normal BCVA $(\log M A R \leq 0$ and Snellen equivalent $\geq 20 / 20$ ) and eyes with decreased BCVA $(\log M A R>0$ and Snellen equivalent $<20 / 20)$. Eyes were also classified into three groups according to the ETDRS level: no or minimal NPDR, level 20; mild NPDR, level 35; moderate or severe NPDR, level 43-53.

\section{OCTA and quantitative analysis of the macular capillary plexuses}

After pupillary dilation, the retinal microvasculature was imaged in a dark room by the RTVue XR Avanti SD-OCT system (Optovue, Inc., Fremont, CA, USA) equipped with AngioVue software (Version 2017.1.0.155). We chose the macular $3 \times 3$-mm program, with a scan speed at 70,000 A-scans per second and pixel dimensions of $304 \times 304$. OCTA images with significant image artifacts 
and poor image quality (signal strength index $<40$ ) were excluded, an example is given in Additional file 1: Fig. S1. The superficial retinal capillary plexus (SRCP) and deep retinal capillary plexus (DRCP) were detected and separated automatically.

A custom automated algorithm was used to quantify the SRCP and DRCP vessel densities in the en face OCTA projection images as described previously [13, 19, 20]. In brief, images were exported from the OCTA device. To enhance the details, the grayscale of each two-dimensional OCTA image was first extended by bicubic interpolation to $1024 \times 1024$ pixels. Then, the image was segmented to obtain the microvascular networks. The vessel density was calculated for the $2.5-\mathrm{mm}$ diameter annular zone after excluding the FAZ (diameter $=0.6 \mathrm{~mm}$, Fig. $1 \mathrm{a}, \mathrm{b})$. The methods above were implemented using MATLAB v. 7.10 (MathWorks, Inv., Natick, MA, USA). The SRCP was defined as a slab extending from the internal limiting membrane to $9 \mu \mathrm{m}$ above the inner plexiform layer (IPL) (Fig. 1c1). The DRCP was defined as a slab extending from $9 \mu \mathrm{m}$ above the IPL to $9 \mu \mathrm{m}$ below the OPL (Fig. 1c2). The FAZ area was calculated using the area function on the integrated automated algorithms 2.0 software.
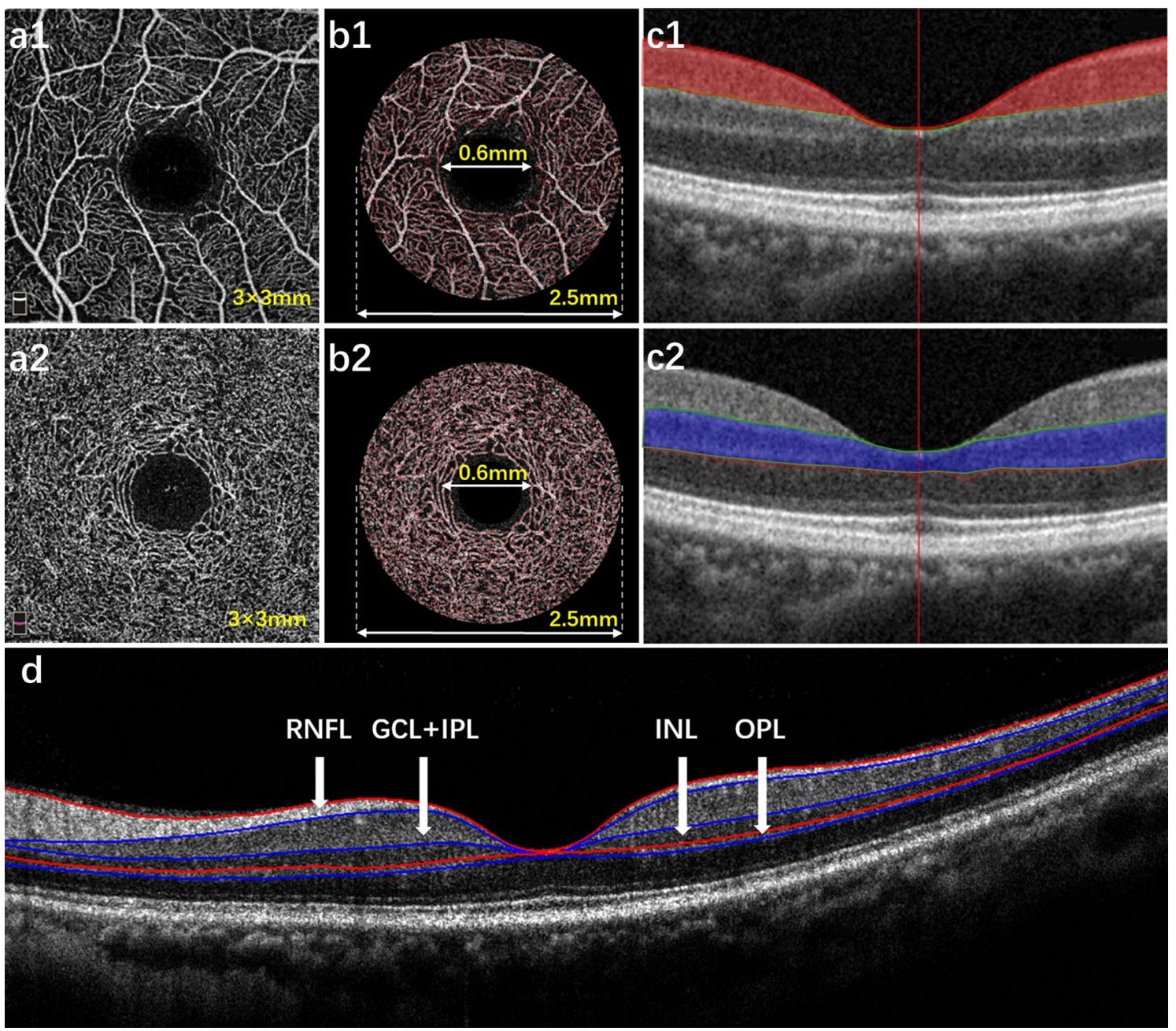

Fig. 1 Optical coherence tomography angiography (OCTA) images showing vessel density analysis of the superficial and deep retinal capillary plexuses (SRCP and DRCP) and the intraretinal layers analyzed by custom software. OCTA images of the SRCP (a1) and DRCP (a2) in the $3 \times 3$-mm area around the fovea. Binary images of the microvascular network in the SRCP (b1) and DRCP (b2) showing the density in the annular zone with a diameter of $2.5 \mathrm{~mm}$ after excluding the foveal avascular zone (diameter $=0.6 \mathrm{~mm}$ ). $\mathbf{c 1}$ The SRCP (red) is shown as a slab extending from the internal limiting membrane to $9 \mu \mathrm{m}$ above inner plexiform layer (IPL). c2 The DRCP (blue) is shown as a slab extending from $9 \mu \mathrm{m}$ above IPL to $9 \mu \mathrm{m}$ below outer plexiform layer. $\mathbf{d}$ Intraretinal layer structures in horizontal scan OCT images: RNFL, retinal nerve fiber layer; GCL-IPL, ganglion cell layer plus inner plexiform layer; INL, inner nuclear layer; OPL, outer plexiform layer 


\section{SD-OCT measurement of individual retinal layer} thicknesses

As described previously [11], each cross-sectional image from the "radial" mode of the OCT scans was analyzed by one masked reader (JL) to segment the boundaries of the intraretinal layers, and the average thickness was calculated. Automatic segmentation was achieved by a custom software program based on the gradient information and shortest path search that was developed in MATLAB for image analysis (Fig. 1d). Each image was manually confirmed through visual inspection by a masked grader after automatic segmentation. The central retinal thickness was defined as the average thickness $(\mu \mathrm{m})$ in the central $1-\mathrm{mm}$ circle defined by ETDRS. The central retinal thickness of the INL, OPL, and total retina were used to evaluate subclinical edema. Neurodegenerative changes were estimated based on RNFL and GCL-IPL average thicknesses in a central 6-mm circle.

\section{Statistical analysis}

Data analyses were performed using the SPSS software (SPSS v.22.0, Chicago, IL). The t-test, ANOVA or Kruskal-Wallis test (for continuous variables) and $X^{2}$ test (for categorical variables) were used to analyze differences between each cohort. The mean and standard deviation (SD) values of the healthy control group served as reference values. Changes were considered to be obvious if different from normal values by more than 1.96 SD. Univariate linear regression models were fit using age, DM duration, ETDRS level, eye, vessel density, FAZ area, and intraretinal or total layer thickness as a single predictor, with BCVA as the outcome. Results from univariate regression models were then used to create a multivariate model with BCVA as the outcome. Parameters that were statistically significant were used to construct the final multivariate regression model and evaluated for the presence of any interactions. To adjust the inter-eye correlation from the same participant, as some patients had bilateral imaging, and to consider possible different demographic characteristics, the generalized estimating equations (GEE) method was used throughout the analysis whenever applicable. A value of $P$ less than 0.05 was considered statistically significant.

\section{Results}

\section{Subject data collection}

Eyes without DR $(\mathrm{n}=25)$, with diabetic macular edema $(n=9)$, proliferative diabetic retinopathy $(n=1)$, or history of treatment $(n=3)$ were excluded. Among the 89 subjects with type 2 diabetes, 145 eyes were potentially eligible for this study. After quality checks, only 132 eyes were included in the final analysis. Of the 89 diabetic patients (132 eyes), 53 patients (88 eyes, 66.7\%) had a normal BCVA with a mean (SD) BCVA of -0.04 (0.06) $\log M A R$. Thirty-six patients (44 eyes, 33.3\%) had decreased BCVA with a BCVA of 0.12 (0.14) logMAR. Table 1 summarizes the demographic and ocular findings of the groups with normal and decreased BCVA.

\section{Different disease pathways in diabetic retinopathy Ischemia}

Ischemia, represented by decreased vessel density and enlargement of the FAZ, was present in $19.3 \%$ of DR eyes with normal BCVA and $47.7 \%$ of DR eyes with decreased BCVA $(P=0.001$, Table 2$)$. It was also present in $14.3 \%$, $25.0 \%$, and $70.8 \%$ of the eyes in ETDRS groups level 20, 35 , and 43-53, respectively $(P<0.001$, Table 2$)$. After adjusting for confounding factors of age and DM duration, there were significant differences in vessel density in SRCP, DRCP, and the FAZ area among the three groups (Table 3). The DRCP vessel density was decreased in DR patients with decreased BCVA compared to DR patients with normal BCVA (0.062 vs. $0.069, P<0.001$, Table 3 , Fig. 2a). Neither the vessel density in the SRCP nor the FAZ area differed significantly between patients with and without decreased BCVA changes (Table 3).

\section{Neurodegeneration}

For DR eyes with normal BCVA, there was no detectable thinning of the RNFL or GCL-IPL (Table 2). In contrast, for eyes with decreased BCVA, $11.4 \%$ had neurodegenerative changes $(P=0.004$ vs. eyes with normal BCVA). Neurodegeneration, represented by thinning of the RNFL and GCL-IPL, was present in $3.6 \%, 3.8 \%, 4.2 \%$ of the eyes in ETDRS groups level 20, 35, and 43-53, respectively $(P=1.000$, Table 2$)$. After adjusting for age and the duration of DM, there were no detectable differences in the thickness of the RNFL among the control, DR with normal BCVA, and DR with decreased BCVA groups $(P>0.05$, Table 3$)$. The thickness of the GCL-IPL in eyes with normal BCVA, $73.62 \mu \mathrm{m}$, was greater than in eyes with decreased BCVA, 70.24 $\mu \mathrm{m}(P=0.033$, Table 3, Fig. 2b). Therefore, we found that most of the neurodegenerative changes took place in the GCL-IPL.

\section{Subclinical edema}

Subclinical edema, indicated by increased retinal thickness, was present in $18.2 \%$ of the DR eyes with normal BCVA and $15.9 \%$ of the DR eyes with decreased BCVA $(P=0.746$, Table 2$)$. It was present in $19.6 \%, 9.6 \%$, and $29.2 \%$ of the eyes in ETDRS groups level 20, 35, and $43-53$, respectively $(P=0.094$, Table 2$)$. After adjusting for age and duration of DM, the thicknesses of the INL, OPL, and total retina were similar among the three groups $(P>0.05$ for all, Table 3$)$. 
Table 1 Control and diabetic retinopathy population and ocular characteristics

\begin{tabular}{|c|c|c|c|c|c|c|c|}
\hline & \multirow[t]{2}{*}{ Control } & \multirow[t]{2}{*}{ DR } & \multirow{2}{*}{$\begin{array}{l}\text { DR with normal } \\
\text { BCVA (G1) }\end{array}$} & \multirow{2}{*}{$\begin{array}{l}\text { DR with } \\
\text { decreased BCVA } \\
\text { (G2) }\end{array}$} & \multicolumn{3}{|l|}{$P$ value } \\
\hline & & & & & All groups & Control vs. DR & G1 vs. G2 \\
\hline \multicolumn{8}{|c|}{ Population characteristics $(n=89)$} \\
\hline No. of participants, $n$ & 33 & 89 & 53 & 36 & NA & NA & NA \\
\hline Age (years) & $56.94 \pm 7.32$ & $54.66 \pm 10.95$ & $50.72 \pm 9.43$ & $60.47 \pm 10.55$ & $<0.001^{\mathrm{a}}$ & $0.960^{\mathrm{a}}$ & $<0.001^{\mathrm{a}}$ \\
\hline $\operatorname{Sex}(M / F)$ & $12 / 21$ & $54 / 35$ & $36 / 17$ & $18 / 18$ & $0.014^{b}$ & $0.017^{b}$ & $0.089^{b}$ \\
\hline $\mathrm{BMI}\left(\mathrm{kg} / \mathrm{m}^{2}\right)$ & $24.57 \pm 2.49$ & $25.40 \pm 3.67$ & $25.56 \pm 3.35$ & $25.16 \pm 4.15$ & $0.433^{\mathrm{a}}$ & $0.452^{\mathrm{a}}$ & $0.598^{\mathrm{a}}$ \\
\hline MAP (mmHg) & $92.42 \pm 10.61$ & $96.41 \pm 22.61$ & $96.78 \pm 24.11$ & $95.82 \pm 20.29$ & $0.079^{c}$ & $0.372^{c}$ & $1.000^{c}$ \\
\hline DM duration (years) & NA & $9.06 \pm 6.34$ & $7.62 \pm 5.27$ & $11.19 \pm 7.21$ & NA & NA & $0.021^{c}$ \\
\hline $\mathrm{BG}(\mathrm{mmol} / \mathrm{L})$ & NA & $7.78 \pm 2.64$ & $7.42 \pm 2.46$ & $8.35 \pm 2.83$ & NA & NA & $0.067^{\mathrm{a}}$ \\
\hline \multicolumn{8}{|c|}{ Ocular characteristics $(n=132)$} \\
\hline No. of eyes, $n$ & 33 & 132 & 88 & 44 & NA & NA & NA \\
\hline BCVA (logMAR) & $-0.05 \pm 0.05$ & $0.01 \pm 0.12$ & $-0.05 \pm 0.06$ & $0.12 \pm 0.14$ & $<0.001^{c}$ & $0.008^{c}$ & $<0.001^{c}$ \\
\hline SE (D) & $0.16 \pm 1.16$ & $-0.10 \pm 1.39$ & $-0.13 \pm 1.42$ & $0.21 \pm 1.27$ & $0.009^{c}$ & $0.071^{c}$ & $0.038^{c}$ \\
\hline $\mathrm{IOP}(\mathrm{mmHg})$ & $12.53 \pm 2.42$ & $14.41 \pm 3.48$ & $14.20 \pm 3.53$ & $14.83 \pm 3.37$ & $0.012^{\mathrm{a}}$ & $0.005^{\mathrm{a}}$ & $0.306^{\mathrm{a}}$ \\
\hline \multicolumn{8}{|l|}{ DR severity } \\
\hline ETDRS $20(n=56)$ & NA & 56 & 39 & 17 & NA & NA & $0.534^{b}$ \\
\hline ETDRS $35(n=52)$ & NA & 52 & 33 & 19 & NA & NA & $0.529^{b}$ \\
\hline ETDRS $43-53(n=24)$ & NA & 24 & 16 & 8 & NA & NA & $1.000^{b}$ \\
\hline
\end{tabular}

BCVA best-corrected visual acuity; NA not applicable; $M / F$ male/female; $B M /$ body mass index; $M A P$ mean arterial pressure; $D M$ diabetes mellitus; $B G$ blood glucose; logMAR logarithm of the minimum angle of resolution; IOP intraocular pressure; DR diabetic retinopathy; ETDRS Early Treatment Diabetic Retinopathy

a t-test or ANOVA

${ }^{\mathrm{b}} \mathrm{X}^{2}$ test

${ }^{\mathrm{c}}$ Kruskal-Wallis test

\section{Overall distribution}

In DR eyes with normal BCVA, 65\% did not have any obvious alteration in either ischemia, neurodegeneration, or subclinical edema. Among the DR subjects with normal BCVA eyes, $17 \%$ and $16 \%$ showed a single pattern of ischemia and subclinical edema, respectively, and $2 \%$ had both pathological manifestations. However, in DR eyes with decreased BCVA, only $43 \%$ had no detectable vascular or neurodegenerative changes (Fig. 3). Among the eyes with detectable changes, $39 \%$ had a single pathological mechanism, of which $30 \%$ had ischemia, $4 \%$ had neurodegeneration, and $5 \%$ had subclinical edema. Interestingly, some patients had two different pathological indicators. Thus, $11 \%$ of the eyes with reduced BCVA had both ischemia and subclinical edema, and $7 \%$ had ischemic and neurodegeneration simultaneously.

\section{Associations of different disease pathways with visual acuity in DR}

Univariate GEE analyses showed that age $(P<0.001)$, duration of DM $(P=0.011)$, DRCP vessel density $(P=0.002)$, FAZ area $(P=0.022)$, and GCL-IPL thickness $(P<0.001)$ were significantly associated with BCVA. ETDRS level, eye, SRCP vessel density, and thickness of the RNFL, INL, OPL, and total retinal were not associated with BCVA (all $P \geq 0.05$, Table 4). The significant predictors of BCVA in the univariate GEE analyses were then used in the final predictive model. Age, DRCP vessel density, and GCL-IPL thickness remained as significant predictors of BCVA in the final model (Table 4). Analysis of the receiver operating characteristics was used to calculate the cutoff value that determined the presence of visual impairment, defined as BCVA worse than 20/20 (Additional file 2: Table S1). Based on multiple regression models that affected the BCVA outcome, we explored the interactions between the GCL-IPL thickness and DRCP vessel density with BCVA as the outcome (Table 5), and the interaction was observed to be significant $(P<0.001)$.

\section{Discussion}

We utilized OCT- and OCTA-derived anatomic and microvascular parameters to investigate the visual significance of three different pathological pathways, i.e., ischemia, neurodegeneration, and subclinical edema in the natural progression of the early stages of DR. We then determined if these factors mutually influenced vison loss. The major findings of this study are as follows: (1) the prevailing mechanism of visual acuity loss may be different in different patients at the initial stage 


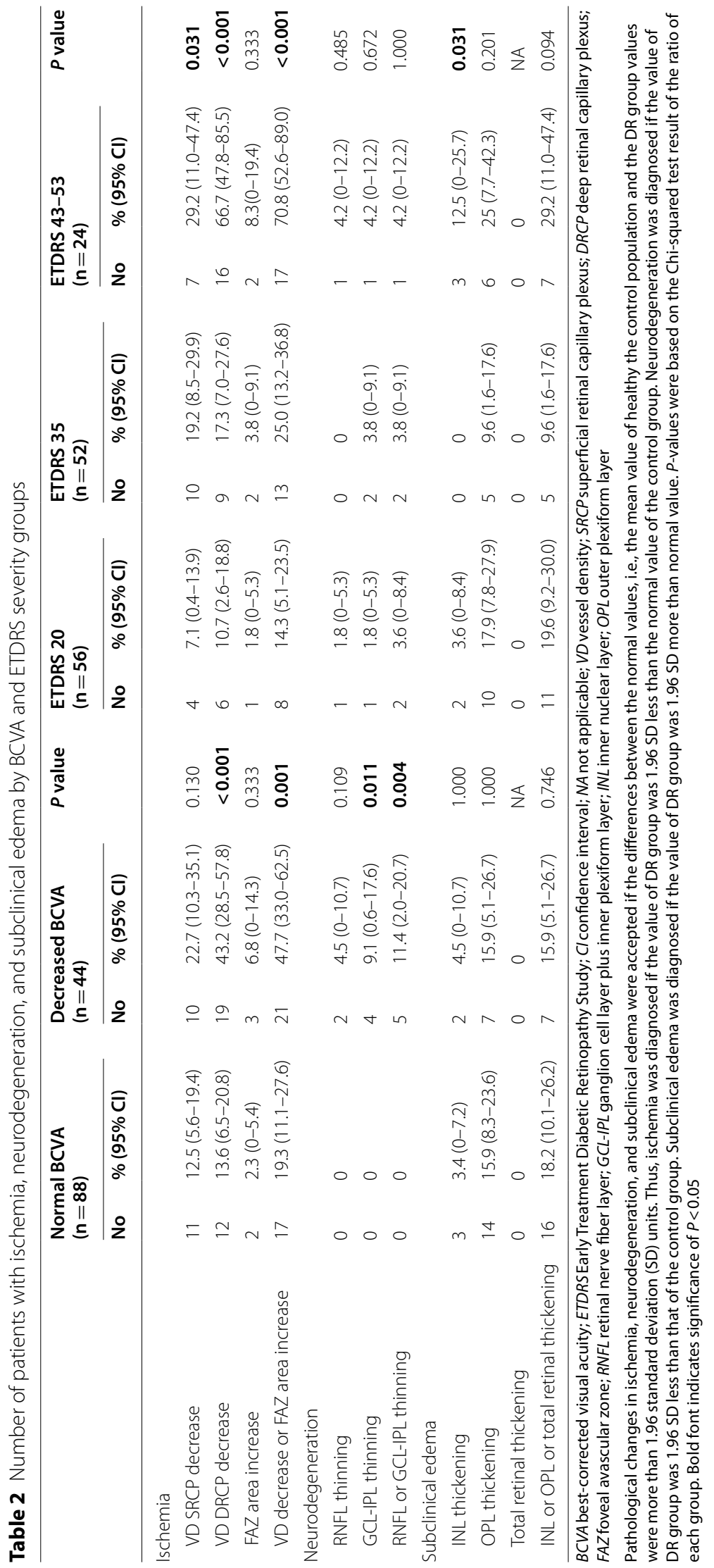


Table 3 Comparison of retinal microvascular parameters and retinal sublayer or total thickness in control and diabetic groups after adjusting for age, duration of DM

\begin{tabular}{|c|c|c|c|c|c|c|c|}
\hline & Control (G0) & $\begin{array}{l}\text { DR With } \\
\text { normal BCVA } \\
\text { (G1) }\end{array}$ & $\begin{array}{l}\text { DR with } \\
\text { decreased BCVA } \\
\text { (G2) }\end{array}$ & $P$ All groups & PG0 vs. G1 & PG0 vs. G2 & PG1 vs. G2 \\
\hline \multicolumn{8}{|l|}{ Microvascular parameters } \\
\hline VD SRCP & $0.056 \pm 0.004$ & $0.054 \pm 0.005$ & $0.052 \pm 0.006$ & 0.004 & 0.038 & 0.002 & 0.128 \\
\hline VD DRCP & $0.072 \pm 0.005$ & $0.069 \pm 0.006$ & $0.062 \pm 0.007$ & $<0.001$ & 0.007 & $<0.001$ & $<0.001$ \\
\hline FAZ area $\left(\mathrm{mm}^{2}\right)$ & $0.325 \pm 0.132$ & $0.342 \pm 0.117$ & $0.401 \pm 0.097$ & 0.019 & 0.466 & 0.008 & 0.196 \\
\hline \multicolumn{8}{|l|}{ Retinal layer thickness } \\
\hline RNFL thickness ( $\mu \mathrm{m})$ & $28.46 \pm 2.88$ & $30.04 \pm 3.14$ & $29.04 \pm 3.64$ & 0.214 & 0.082 & 0.272 & 0.849 \\
\hline GCL-IPL thickness $(\mu \mathrm{m})$ & $72.40 \pm 5.59$ & $73.62 \pm 4.82$ & $70.24 \pm 6.21$ & 0.112 & 0.653 & 0.182 & 0.033 \\
\hline INL thickness ( $\mu \mathrm{m})$ & $17.52 \pm 3.66$ & $17.10 \pm 3.41$ & $17.10 \pm 3.82$ & 0.852 & 0.706 & 0.572 & 0.943 \\
\hline OPL thickness $(\mu \mathrm{m})$ & $10.15 \pm 1.49$ & $10.89 \pm 2.51$ & $10.60 \pm 2.36$ & 0.240 & 0.108 & 0.217 & 0.666 \\
\hline Total retinal thickness $(\mu \mathrm{m})$ & $239.46 \pm 21.01$ & $240.69 \pm 16.13$ & $236.31 \pm 19.14$ & 0.663 & 0.866 & 0.538 & 0.680 \\
\hline
\end{tabular}

$D M$ diabetes mellitus; $B C V A$ best-corrected visual acuity; $S R C P$ superficial retinal capillary plexus; $D R C P$ deep retinal capillary plexus; $V D$ vessel density; $F A Z$ foveal avascular zone; RNFL retinal nerve fiber layer; GCL-IPL ganglion cell layer plus inner plexiform layer; INL inner nuclear layer; OPL outer plexiform layer Bold font indicates significance of $P<0.05$
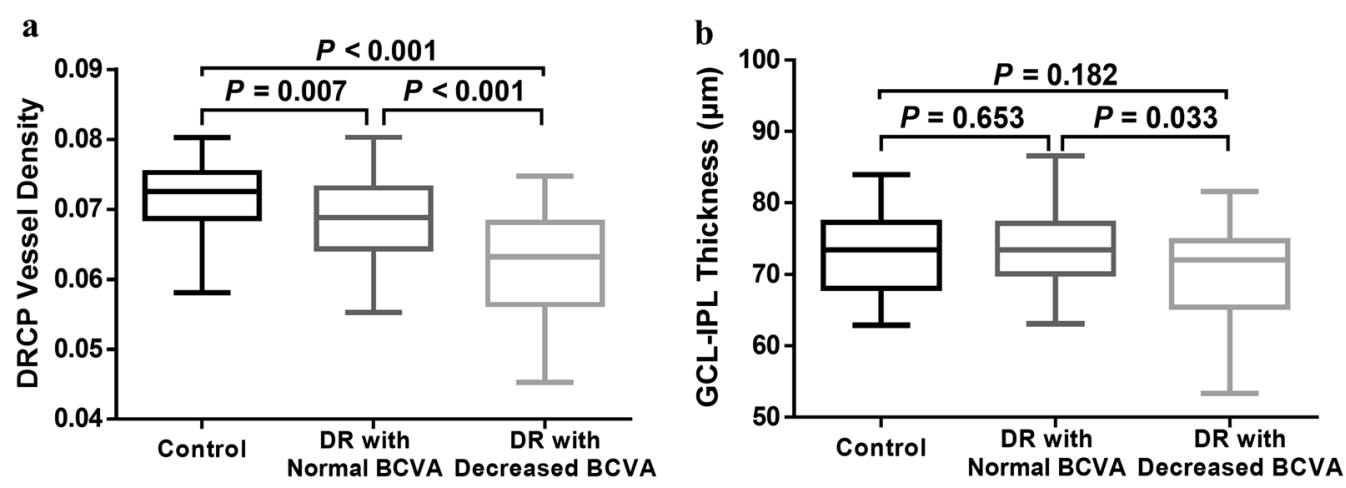

Fig. 2 Comparison of deep retinal capillary plexus (DRCP) vessel density and ganglion cell layer plus inner plexiform layer (GCL-IPL) thickness between control group, diabetic retinopathy (DR) eyes with normal best-corrected visual acuity (BCVA) and DR eyes with decreased BCVA. The vessel density in DRCP (a) and thickness of GCL-IPL (b) are significantly lower in eyes with DR and decreased BCVA than in eyes with DR and normal BCVA

of DR; (2) we found that both ischemia, evaluated by OCTA-documented loss of vessel density in the DRCP, and neurodegeneration, evaluated by OCT-documented thinning of the GCL-IPL, were independently correlated with decreases in BCVA; (3) ischemia and neurodegeneration mutually influenced each other in affecting vision loss. Therefore, preventing further neurodegeneration as well as ischemia should be an important clinical goal for applying precision medicine in early diabetic retinal disease.

Our findings may contribute to the individual management of DR in the context of preventing early threats to vision. In our study, some DR eyes were found to manifest a single phenotype, i.e., ischemia or neurodegenerative. Interestingly, a portion had overlapping ischemia and neurodegeneration or ischemia and subclinical edema. These changes suggest that the predominant mechanism of visual impairment may be different in different patients, and indicators based on OCT and OCTA may help identify factors that threaten a patient's vision, thereby guiding us to optimize the individualized treatment methods. Further longitudinal studies are required to determine if a personalized assessment of these pathways would protect the BCVA during the development of DR.

In this study, we found that of the three different disease pathways, ischemia was the main factor that threatened vision in the early stages of DR, which is in concordance with the conclusions of other reports [21-24]. In addition to ischemia, we discovered that neurodegeneration 
a Normal BCVA

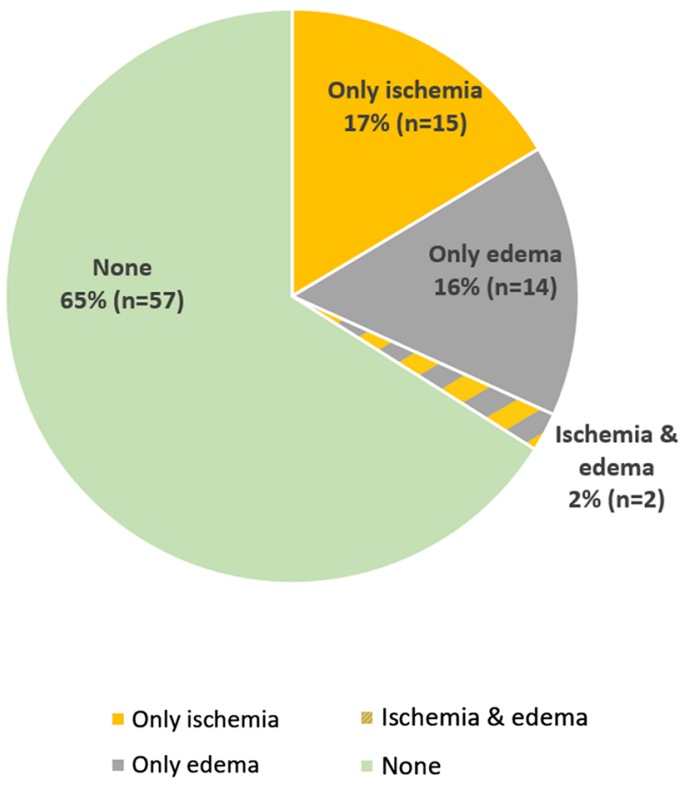

b Decreased BCVA

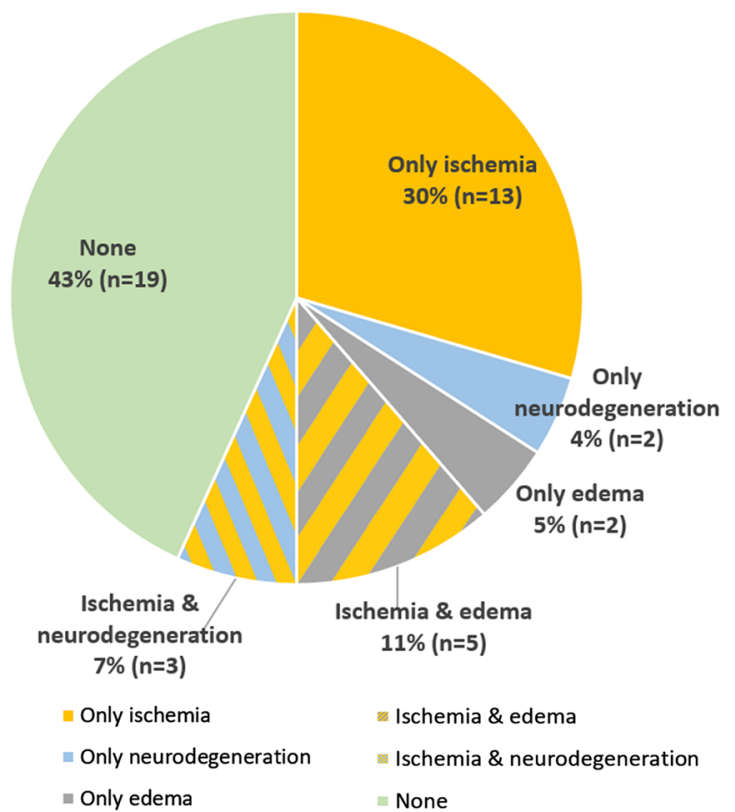

Fig. 3 Distribution of eyes with different pathological conditions in diabetic retinopathy (DR) with normal best-corrected visual acuity (BCVA) and DR with decreased BCVA groups. Eyes with only one lesion appear as one color, and two lesions appear as two mixed colors

Table 4 Results of simple and multiple regression models based on best corrected visual acuity outcome

\begin{tabular}{|c|c|c|c|c|c|c|c|c|}
\hline \multirow{2}{*}{ Parameters } & \multicolumn{4}{|c|}{ Simple regression model } & \multicolumn{4}{|c|}{ Multiple regression model } \\
\hline & Coefficient & Standard error & $95 \% \mathrm{Cl}$ & $P$ value & Coefficient & Standard error & $95 \% \mathrm{Cl}$ & $P$ value \\
\hline Age & 0.003 & 0.001 & 0.001 to 0.005 & $<0.001$ & 0.002 & 0.001 & 0.001 to 0.004 & 0.009 \\
\hline DM duration & 0.004 & 0.002 & 0.001 to 0.007 & 0.011 & - & - & - & - \\
\hline ETDRS level & 0.002 & 0.001 & -0.0003 to 0.004 & 0.087 & - & - & - & - \\
\hline Eye & 0.007 & 0.017 & -0.027 to 0.041 & 0.686 & - & - & - & - \\
\hline VD SRCP & -3.906 & 1.993 & -7.813 to 0.001 & 0.050 & - & - & - & - \\
\hline VD DRCP & -7.258 & 2.344 & -11.852 to -2.663 & 0.002 & -6.194 & 2.605 & -11.298 to -1.089 & 0.017 \\
\hline FAZ area & 0.448 & 0.195 & 0.065 to 0.830 & 0.022 & - & - & - & - \\
\hline RNFL thickness & -0.005 & 0.003 & -0.012 to 0.001 & 0.120 & - & - & - & - \\
\hline GCL-IPL thickness & -0.007 & 0.002 & -0.010 to -0.004 & $<0.001$ & -0.004 & 0.002 & -0.007 to -0.001 & 0.044 \\
\hline INL thickness & -0.0005 & 0.003 & -0.006 to 0.005 & 0.856 & - & - & - & - \\
\hline OPL thickness & -0.001 & 0.005 & -0.010 to 0.009 & 0.912 & - & - & - & - \\
\hline Total thickness & -0.002 & 0.001 & -0.004 to 0.0002 & 0.079 & - & - & - & - \\
\hline Constant & NA & NA & NA & NA & 0.575 & 0.196 & 0.191 to 0.959 & 0.003 \\
\hline
\end{tabular}

Cl confidence interval; DM diabetes mellitus; ETDRSEarly Treatment Diabetic Retinopathy Study; VDvessel density; SRCP superficial retinal capillary plexus; $D R C P$ deep retinal capillary plexus; FAZfoveal avascular zone; RNFL retinal nerve fiber layer; GCL-IPL ganglion cell layer plus inner plexiform layer; INL inner nuclear layer; OPL outer plexiform layer; $N A$ not applicable

Bold font indicates significance of $P<0.05$

also plays a huge role in the loss of BCVA even in the early stages of DR. Further, we found that the thickness of the GCL-IPL was a more sensitive biomarker of early DR visual changes than RNFL. Reductions in the neurological layers are likely to indicate a reduced abundance of retinal ganglion cell axons, and probably a loss of cell bodies and dendrites. This defect may become an obstacle to the transmission of visual information to the 
Table 5 Multiple regression models for interactions between the GCL-IPL thickness and deep retinal capillary plexus vessel density based on best corrected visual acuity outcome

\begin{tabular}{lllll}
\hline Parameters & Coefficient & $\begin{array}{l}\text { Standard } \\
\text { error }\end{array}$ & $\mathbf{9 5 \%} \mathrm{Cl}$ & $\boldsymbol{P}$ value \\
\hline $\begin{array}{l}\text { GCL-IPL thick- } \\
\text { neSS } \times \text { VD }\end{array}$ & -0.083 & 0.020 & $\begin{array}{c}-0.121 \\
\text { to }-0.144\end{array}$ & $<0.001$ \\
DRCP & & & & \\
\hline
\end{tabular}

GCL-IPL ganglion cell layer plus inner plexiform layer; VDvessel density; $D R C P$ deep retinal capillary plexus

brain and damage the information processing capabilities of the inner retina. Several studies also showed the early neurodegeneration in diabetic patients [25-27]. Alteration in retinal trophic factors, oxidative stress, and mitochondrial damage induced by hyperglycemia, low-grade inflammation, immune cell activation, and extracellular glutamate accumulation are crucial for the development of retinal neurodegeneration [28, 29]. However, the decrease of GCL-IPL thickness was not so evident and the casual relationships between neurodegeneration and BCVA decline was unclear in the current study. We assume that neurodegeneration may be more related to other visual function defects such as electroretinography, microperimetry, contrast sensitivity, and color vision, all of which could be evaluated in future studies.

In this report, we grouped patients using two different criteria, i.e., BCVA and ETDRS. We found that the distribution patterns of the three pathological mechanisms in the two classifications were significantly different. Only ischemia was correlated with disease severity as evaluated by the ETDRS criteria, and neurodegeneration were evenly distributed among ETDRS groups, which are in tandem with previous findings [30]. However, our results showed that neurodegeneration was an independent predictor of BCVA. Therefore, solely evaluating diabetic patients by vascular manifestation may lead to the risk that visual impairment could go undetected.

DR classification schemes have been extremely useful because they were designed in an era when the most essential issue was dealing with severe blinding retinopathy. The success of the classification schemes now brings us to the twenty-first century in which a consensus has been reached that treatment of DR should begin prior to the onset of vision-threatening stages [31, 32]. However, the conventional classification is based primarily on microvascular changes and does not incorporate recent findings of structural neuropathy in diabetes. Hence, the development of a new and comprehensive classification system of DR has been proposed [2]. Our results have provided fundamental evidence that monitoring neurodegeneration is of value for clinical endpoints and should be considered in the new and clinically useful classification scheme.

While both ischemia and neurodegeneration were independently correlated with decreased BCVA, they also interacted together to increase vision loss. Neurodegeneration may precede microvascular dysfunction in DR, and it may contribute to microvascular abnormalities [33]. The neurovascular unit may serve as the connection that links neurons and capillaries, and it may be the anatomical basis for the mutual influence of neurodegeneration and ischemia [34]. However, a more definitive understanding of the mutual influence of the two mechanisms needs to be clarified in future research.

It is a finding of interest that the OCT-measured subclinical edema, identified by retinal thickening compared to the normal control group, was not correlated with BCVA. Generally, diabetic macular edema is one of the main reasons that affect visual acuity of diabetic patients. However, subclinical edema was not related to BCVA, indicating timely control of edema in the subclinical course may prevent the occurrence of potential severe visual impairment. This hypothesis could be further verified in future studies.

We acknowledge several limitations of this study. First, the casual relationships between ischemia and neurodegeneration and the decrease of the BCVA were unclear due to the cross-sectional nature of the study and the limited number of subjects; thus, longitudinal studies of larger sample sizes are required to validate the findings. Second, we checked all OCT images and found no significant structural damage in the retinal layers. Nevertheless, some parameters such as ellipsoid zone disruption and disorganization of the retinal inner layers and the intercapillary area have been reported to be associated with loss of BCVA in DR. These potential factors, along with clinical characteristics such as axial length and $\mathrm{HbA} 1 \mathrm{c}$ that could alter OCT and OCTA parameters and affect BCVA, should be considered in the future studies [35-37]. Third, although OCTA provided improved visualization of the microvasculature in different retinal layers compared to the traditional fundus images, especially in the early DR patients, it has limitations such as a small field of view in $3 \times 3 \mathrm{~mm}$, the influence of scan quality, and the inability to show the leakage. It is worth mentioning that fluorescein angiography is unsurpassed in the assessment of macular ischemia. Fluorescein angiography can often detect DR in patients that have no apparent DR on the basis of 7-field protocol using the ETDRS classification. Fourth, to minimize the impact of lens opacities on the decline of BCVA, we made a subjective assessment of lens status based upon expert analysis of slit-lamp 
biomicroscopy images, and only OCTA images with a signal strength index greater than 40 were chosen. The lack of lens status information that could have affected visual acuity may have confounded our findings.

\section{Conclusions}

In conclusion, we demonstrated that subtle alterations in the microvasculature and neuroretina of DR eyes associated with decreased visual acuity can be detected quantitively by OCTA and OCT. Ischemia and neurodegeneration are critical factors that are related to the visual impairment and could exert a mutual influence over the natural course of the early stages of DR. These changes constitute prominent pathophysiological mechanisms in early DR, but they vary greatly among patients. A multimodal imaging protocol monitoring both microvascular alteration and neurodegenerative change is essential to identify the eyes at a higher risk for future vision loss, which will enhance the development of precision medicine in the management of DR.

\section{Supplementary Information}

The online version contains supplementary material available at https://doi. org/10.1186/s40662-021-00260-4.

Additional file 1: Fig. S1. An example of significant artifact (SSI=35). The yellow arrow shows the location of artifact (a). An example with SSI $=69$ (b) was provided for comparison.

Additional file 2: Table S1. ROC analysis of microvascular parameters and retinal thickness of diabetic patients with normal and decreased BCVA.

\section{Acknowledgements}

The authors thank Prof. Jingwei Zheng for guidance in statistical analysis.

\section{Authors' contributions}

Study concept and design: JL, YZ, FC, YL, RZ, CW, CS, YS, QC, FL MS. Acquisition, analysis, or interpretation of data: JL, YZ, HY, ZL, GZ. Drafting of the manuscript: JL, YZ, MS. Critical revision of the manuscript for intellectual content: all authors. Statistical analysis: JL, YZ. Administrative, technical or material support: FC, YL, RZ, CW, FL, MS. All authors read and approved the final manuscript.

\section{Funding}

This work was supported by the National Key Project of Research and Development Program of Zhejiang Province (2019C03045), National Natural Science Foundation of China (82171016), Wenzhou Municipal Science and Technology Bureau (2018ZY016).

\section{Availability of data and materials}

The datasets used and/or analyzed during the current study are available from the corresponding author on reasonable request.

\section{Declarations}

Ethics approval and consent to participate

This study followed the tenets of the Declaration of Helsinki and was approved by the ethics committee of the Eye Hospital of Wenzhou Medical University (KYK[2017]No.41).
Consent for publication

Not applicable.

\section{Competing interests}

The authors declare that they have no competing interests.

\section{Author details}

${ }^{1}$ School of Ophthalmology and Optometry, Wenzhou Medical University, 270 Xueyuan Road, Wenzhou 325027, Zhejiang, China. ${ }^{2}$ The Second Affiliated Hospital and Yuying Children's Hospital of Wenzhou Medical University, Wenzhou 325027, Zhejiang, China. ${ }^{3}$ Department of Ophthalmology, The Affiliated Hospital of Yangzhou University, Yangzhou 225000, Jiangsu, China.

Received: 25 May 2021 Accepted: 21 September 2021

Published online: 19 October 2021

\section{References}

1. Lee R, Wong TY, Sabanayagam C. Epidemiology of diabetic retinopathy, diabetic macular edema and related vision loss. Eye Vis (Lond). 2015;2:17.

2. Abramoff MD, Fort PE, Han IC, Jayasundera KT, Sohn EH, Gardner TW. Approach for a clinically useful comprehensive classification of vascular and neural aspects of diabetic retinal disease. Invest Ophthalmol Vis Sci. 2018;59(1):519-27.

3. Zafar S, Sachdeva M, Frankfort BJ, Channa R. Retinal neurodegeneration as an early manifestation of diabetic eye disease and potential neuroprotective therapies. Curr Diab Rep. 2019;19(4):17.

4. Marques IP, Alves D, Santos T, Mendes L, Lobo C, Santos AR, et al. Characterization of disease progression in the initial stages of retinopathy in type 2 diabetes: a 2-year longitudinal study. Invest Ophthalmol Vis Sci. 2020;61(3):20.

5. Marques IP, Madeira MH, Messias AL, Martinho AC, Santos T, Sousa DC, et al. Different retinopathy phenotypes in type 2 diabetes predict retinopathy progression. Acta Diabetol. 2021;58(2):197-205.

6. Song P, Yu J, Chan KY, Theodoratou E, Rudan I. Prevalence, risk factors and burden of diabetic retinopathy in China: a systematic review and metaanalysis. J Glob Health. 2018;8(1):010803.

7. Wilkinson CP, Ferris FL 3rd, Klein RE, Lee PP, Agardh CD, Davis M, et al. Proposed international clinical diabetic retinopathy and diabetic macular edema disease severity scales. Ophthalmology. 2003;110(9):1677-82.

8. Joltikov KA, de Castro VM, Davila JR, Anand R, Khan SM, Farbman N, et al. Multidimensional functional and structural evaluation reveals neuroretinal impairment in early diabetic retinopathy. Invest Ophthalmol Vis Sci. 2017;58(6):BIO277-90.

9. Rabiolo A, Cicinelli MV, Corbelli E, Baldin G, Carnevali A, Lattanzio R, et al. Correlation analysis between foveal avascular zone and peripheral ischemic index in diabetic retinopathy: a pilot study. Ophthalmol Retina. 2018;2(1):46-52.

10. Moran EP, Wang Z, Chen J, Sapieha P, Smith LE, Ma JX. Neurovascular cross talk in diabetic retinopathy: pathophysiological roles and therapeutic implications. Am J Physiol Heart Circ Physiol. 2016;311(3):H738-49.

11. Chen Q, Tan F, Wu Y, Zhuang X, Wu C, Zhou Y, et al. Characteristics of retinal structural and microvascular alterations in early type 2 diabetic patients. Invest Ophthalmol Vis Sci. 2018;59(5):2110-8.

12. Ng DS, Chiang PP, Tan G, Cheung CG, Cheng CY, Cheung CY, et al. Retinal ganglion cell neuronal damage in diabetes and diabetic retinopathy. Clin Exp Ophthalmol. 2016;44(4):243-50.

13. Kwapong WR, Ye H, Peng C, Zhuang X, Wang J, Shen M, et al. Retinal microvascular impairment in the early stages of Parkinson's disease. Invest Ophthalmol Vis Sci. 2018;59(10):4115-22.

14. Browning DJ, Fraser CM. The predictive value of patient and eye characteristics on the course of subclinical diabetic macular edema. Am J Ophthalmol. 2008;145(1):149-54.

15. Dimitrova G, Chihara E, Takahashi H, Amano H, Okazaki K. Quantitative retinal optical coherence tomography angiography in patients with diabetes without diabetic retinopathy. Invest Ophthalmol Vis Sci. 2017;58(1):190-6.

16. Durbin MK, An L, Shemonski ND, Soares M, Santos T, Lopes M, et al. Quantification of retinal microvascular density in optical coherence tomographic angiography images in diabetic retinopathy. JAMA Ophthalmol. 2017;135(4):370-6. 
17. Johannesen SK, Viken JN, Vergmann AS, Grauslund J. Optical coherence tomography angiography and microvascular changes in diabetic retinopathy: a systematic review. Acta Ophthalmol. 2019;97(1):7-14.

18. Sim DA, Keane PA, Fung S, Karampelas M, Sadda SR, Fruttiger M, et al. Quantitative analysis of diabetic macular ischemia using optical coherence tomography. Invest Ophthalmol Vis Sci. 2014;55(1):417-23.

19. Kwapong WR, Peng C, He Z, Zhuang X, Shen M, Lu F. Altered macular microvasculature in neuromyelitis optica spectrum disorders. Am J Ophthalmol. 2018;192:47-55.

20. Cheng D, Shen M, Zhuang X, Lin D, Dai M, Chen S, et al. Inner retinal microvasculature damage correlates with outer retinal disruption during remission in Behcet's posterior uveitis by optical coherence tomography angiography. Invest Ophthalmol Vis Sci. 2018;59(3):1295-304.

21. Balaratnasingam C, Inoue M, Ahn S, McCann J, Dhrami-Gavazi E, Yannuzzi $L A$, et al. Visual acuity is correlated with the area of the foveal avascular zone in diabetic retinopathy and retinal vein occlusion. Ophthalmology. 2016;123(11):2352-67.

22. Dupas B, Minvielle W, Bonnin S, Couturier A, Erginay A, Massin P, et al. Association between vessel density and visual acuity in patients with diabetic retinopathy and poorly controlled type 1 diabetes. JAMA Ophthalmol. 2018;136(7):721-8.

23. Samara WA, Shahlaee A, Adam MK, Khan MA, Chiang A, Maguire Jl, et al. Quantification of diabetic macular ischemia using optical coherence tomography angiography and its relationship with visual acuity. Ophthalmology. 2017;124(2):235-44.

24. Sim DA, Keane PA, Zarranz-Ventura J, Fung S, Powner MB, Platteau E, et al. The effects of macular ischemia on visual acuity in diabetic retinopathy. Invest Ophthalmol Vis Sci. 2013;54(3):2353-60.

25. Carpineto P, Toto L, Aloia R, Ciciarelli V, Borrelli E, Vitacolonna E, et al. Neuroretinal alterations in the early stages of diabetic retinopathy in patients with type 2 diabetes mellitus. Eye (Lond). 2016;30(5):673-9.

26. Sohn EH, van Dijk HW, Jiao C, Kok PH, Jeong W, Demirkaya N, et al. Retinal neurodegeneration may precede microvascular changes characteristic of diabetic retinopathy in diabetes mellitus. Proc Natl Acad Sci U S A. 2016;113(19):E2655-64.

27. Chhablani J, Sharma A, Goud A, Peguda HK, Rao HL, Begum VU, et al. Neurodegeneration in type 2 diabetes: evidence from spectral-domain optical coherence tomography. Invest Ophthalmol Vis Sci. 2015;56(11):6333-8.

28. de Moraes $G$, Layton CJ. Therapeutic targeting of diabetic retinal neuropathy as a strategy in preventing diabetic retinopathy. Clin Exp Ophthalmol. 2016;44(9):838-52.

29. Simó R, Stitt AW, Gardner TW. Neurodegeneration in diabetic retinopathy: does it really matter? Diabetologia. 2018;61(9):1902-12.

30. Marques IP, Alves D, Santos T, Mendes L, Santos AR, Lobo C, et al. Multimodal imaging of the initial stages of diabetic retinopathy: different disease pathways in different patients. Diabetes. 2019;68(3):648-53.

31. Nair P, Aiello LP, Gardner TW, Jampol LM, Ferris FL III. Report from the NEI/ FDA diabetic retinopathy clinical trial design and endpoints workshop. Invest Ophthalmol Vis Sci. 2016;57(13):5127-42.

32. Gardner TW, Sundstrom JM. A proposal for early and personalized treatment of diabetic retinopathy based on clinical pathophysiology and molecular phenotyping. Vision Res. 2017;139:153-60.

33. Rossino MG, Dal Monte M, Casini G. Relationships between neurodegeneration and vascular damage in diabetic retinopathy. Front Neurosci. 2019:13:1172

34. Gardner TW, Davila JR. The neurovascular unit and the pathophysiologic basis of diabetic retinopathy. Graefes Arch Clin Exp Ophthalmol. 2017;255(1):1-6.

35. Cheng D, Wang Y, Huang S, Wu Q, Chen Q, Shen M, et al. Macular inner retinal layer thickening and outer retinal layer damage correlate with visual acuity during remission in Behcet's disease. Invest Ophthalmol Vis Sci. 2016;57(13):5470-8.

36. DaCosta J, Bhatia D, Talks J. The use of optical coherence tomography angiography and optical coherence tomography to predict visual acuity in diabetic retinopathy. Eye (Lond). 2020;34(5):942-7.

37. Tang F, Sun Z, Wong R, Lok J, Lam A, Tham CC, et al. Relationship of intercapillary area with visual acuity in diabetes mellitus: an optical coherence tomography angiography study. Br J Ophthalmol. 2018;bjophthalmol-2017-312010. https://doi.org/10.1136/bjophthalmol-2018-312010.

Ready to submit your research? Choose BMC and benefit from:

- fast, convenient online submission

- thorough peer review by experienced researchers in your field

- rapid publication on acceptance

- support for research data, including large and complex data types

- gold Open Access which fosters wider collaboration and increased citations

- maximum visibility for your research: over $100 \mathrm{M}$ website views per year

At BMC, research is always in progress.

Learn more biomedcentral.com/submissions 